\title{
Supermode Control in Integrated Hybrid Si/III-V Optoelectronic Circuits for Modal Gain Enhancement
}

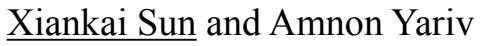 \\ Department of Applied Physics, California Institute of Technology, 1200 E. California Blvd., Pasadena, CA 91125, USA \\ E-mail: xksun@caltech.edu
}

\begin{abstract}
We propose using supermode control to enhance the modal gain in integrated hybrid $\mathrm{Si} / \mathrm{III}-\mathrm{V}$ optoelectronic circuits. Numerical simulations predict that a 4-fold enhancement in modal gain can be achieved with optimal design.
\end{abstract}

Keywords: Optoelectronic integrated circuits; Integrated optics; Si photonics; Semiconductor devices; Modal control.

\section{INTRODUCTION}

The realization of light generation and amplification in silicon ( $\mathrm{Si}$ ) remains an elusive goal in optical communication research. As well known, $\mathrm{Si}$ is an indirect bandgap material thus a very poor convertor of electricity to light. Moreover, due to the crystal centrosymmetry, $2^{\text {nd }}$-order nonlinearities are also highly suppressed in bulk Si. Recent efforts in obtaining optical gain in/on Si include employing Raman amplification [1], doping rare-earth ions [2], engineering nano-crystalline $\mathrm{Si}$ structures [3], and monolithic growth of $\mathrm{Ga}(\mathrm{NAsP})$ via GaP buffer layer [4].

So far the most promising approach is based on a "hybrid evanescent platform" in which an active III-V (AlGaInAs) slab is bonded on top of a pre-patterned Si-on-insulator (SOI) wafer by a low-temperature wafer bonding technique $[5,6]$. Under operation, the bonded structure supports a joint optical mode: the mode is designed to be mostly confined to $\mathrm{Si}$ waveguide and amplified by the penetration of the evanescent tail into the III-V slab. The tradeoff between the confinement factors $(\Gamma)$ in Si waveguide and in III-V slab is the major handicap of this scheme: A high coupling efficiency between the hybrid laser and the rest of the SOI photonic circuit requires that $\Gamma_{\mathrm{Si}}$ be close to 1 , thus reducing $\Gamma_{\mathrm{QW}}$ and the modal gain. Because of the small modal gain, the demonstrated lasers have low emission efficiency compared to traditional III-V lasers.

In this paper we propose controlling the optical mode in such hybrid $\mathrm{Si} / \mathrm{III}-\mathrm{V}$ structures, which eliminates, in principle, the basic compromise inherent in the evanescent laser design since the full modal power, rather than the evanescent tail, is available for amplification. This results in a larger modal gain and increased efficiency.
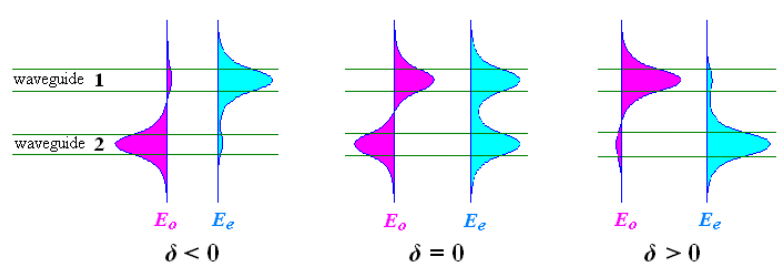

Fig. 1. A schematic representation of the two supermodes $E_{o}$ and $E_{e}$ for three limiting values of the mismatch parameter $\delta$.

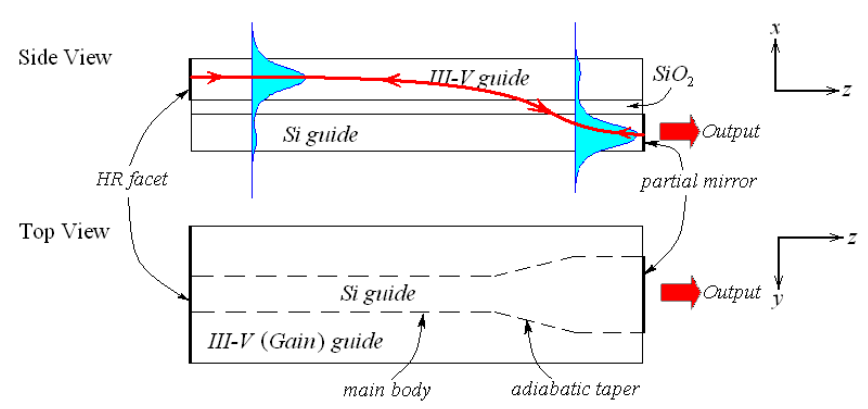

Fig. 2. (Top) Side view of the proposed hybrid laser and the evolution of the lasing supermode inside the hybrid laser resonator. (Bottom) Adiabatic widening of the Si guide causes the supermode power to transfer from the upper amplifying III-V guide to the lower Si guide.

\section{SUPERMODE CONTROL IN SI/III-V HYBRID LASERS}

Our proposed laser utilizes a system of two proximitycoupled waveguides (waveguides $\mathbf{1}$ and 2, as shown in Fig. 1). The first guide is made of a III-V amplifying material while the second is a Si guide fabricated in the SOI substrate. The "supermode" refers to the local eigenmode of this coupled-waveguide system along its propagational direction, which can be expressed as column vectors with their components being the amplitudes of the two individual waveguide modes [7]

$$
E_{e}(z)=\frac{1}{\sqrt{2}}\left|\begin{array}{l}
(1-\delta / S)^{1 / 2} \\
(1+\delta / S)^{1 / 2}
\end{array}\right| e^{-i \beta_{e} z}, E_{o}(z)=\frac{1}{\sqrt{2}}\left|\begin{array}{c}
-(1+\delta / S)^{1 / 2} \\
(1-\delta / S)^{1 / 2}
\end{array}\right| e^{-i \beta_{o} z}
$$

where $\delta=\left(\beta_{2}-\beta_{1}\right) / 2$ is the mismatch of propagation constants between the individual uncoupled waveguide modes, $2 S=$ 


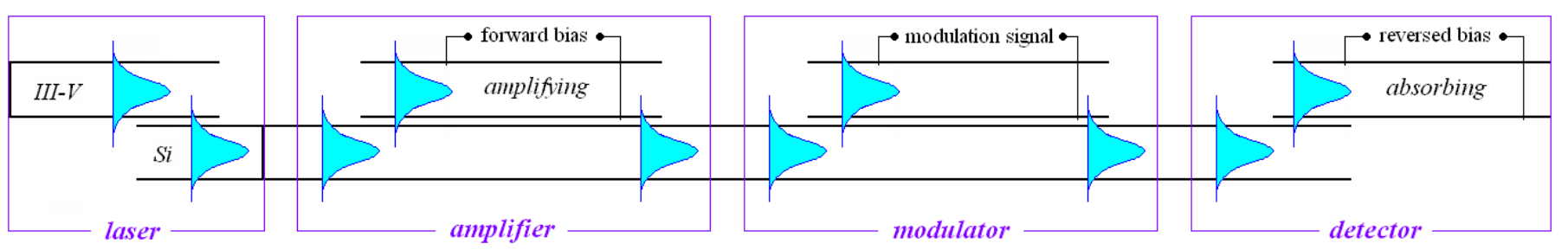

Fig. 3. Conception of future large-scale, high-efficiency hybrid optoelectronic circuitry. Devices with different optical functions can be integrated on the Si platform. The light transportation from one module to another can be on-chip by Si guide or chip-to-chip by optical fibers.

$2\left(\delta^{2}+\kappa^{2}\right)^{1 / 2}=\beta_{e}-\beta_{o}$ is the difference of propagation constants of the supermodes, and $\kappa$ is the coupling strength between the two waveguides. Note that the modal profiles $E_{e}$ and $E_{o}$ are space dependent via the parameters $\delta$ and $S$. For both supermodes, when $\delta<0$ and $|\delta| \gg \kappa$, the mode substantially resides in one waveguide, and when $\delta>0$ and $\delta \gg \kappa$, the mode is substantially in the other waveguide. At the phase-matching point where $\delta=0$ the mode is evenly distributed in the two waveguides [7].

Therefore, as shown in Fig. 2, it is possible, by a proper choice of the width of the Si waveguide, to direct the modal power to the amplifying III-V waveguide or to the $\mathrm{Si}$ waveguide, thereby avoiding the degraded performance which results from the reliance on the evanescent field. The modal power is then diverted to the $\mathrm{Si}$ waveguide by adiabatically increasing its width. This makes for efficient coupling to the outside Si photonic circuit.

As marked with the red line in Fig. 2, the mode starts propagating from left to right in the upper left III-V waveguide where it is amplified. It then enters the adiabatic transformer section where the width of the $\mathrm{Si}$ waveguide increases so as to cause $\delta$ to change from $\delta<0$ to $\delta>0$. This causes the modal energy to shift to the lower low-loss $\mathrm{Si}$ waveguide where it is partially reflected from the right output facet. The reflected field retraces its path till reflected from the upper left facet thus completing the round trip.

A considerable enhancement of modal gain is the most direct advantage of the supermode control. Assuming the identical layer structure used in [5], we have simulated the confinement factors, for the even mode, in the active region (including quantum wells and barriers in between), $\Gamma_{\text {act }}$, and in the Si guide, $\Gamma_{\mathrm{Si}}$, for different $\mathrm{Si}$ guide widths. By evanescent coupling there exists a tradeoff between $\Gamma_{\text {act }}$ and $\Gamma_{\mathrm{Si}}$. A fixed Si guide width of $1.10 \mu \mathrm{m}$ gives $\Gamma_{\mathrm{act}}=0.067$ and $\Gamma_{\mathrm{Si}}=0.757$. By our proposed supermode control, if we vary the Si guide width near the output facet from at main body $0.75 \mu \mathrm{m}$ to at the output facet $1.35 \mu \mathrm{m}$, we will have at main body $\Gamma_{\text {act }}=$ 0.268 , and at the output facet $\Gamma_{\mathrm{Si}}=0.892$. Therefore $\Gamma_{\text {act, }}$, hence the modal gain, increases from 0.067 to 0.268 , i.e., a factor of 4 . In the meantime the output coupling efficiency is also enhanced by, a less impressive, $18 \%$.

\section{Perspective of NeXt-Generation "SuPERmode SI/III-V HYBRID CIRCUITRY"}

The main advantage of this "spatial switching" of the modal power is to obtain maximum achievable gain in the inverted medium since the peak modal field and not just an evanescent tail induce the amplifying transitions in the gain (III-V) region. The same principle of spatial switching of the modal power can be used to achieve other functions. For example: To make an optical amplifier, we can switch the input modal power from a wide $\mathrm{Si}$ waveguide $(\delta>0)$ to a III-V amplifying section $(\delta<0)$ and back again to the wide Si waveguide $(\delta>0)$. This principle can also be applied to make a modulator or a photodetector.

It is envisioned that once the individual optical functional devices are achieved in a much more efficient fashion by using the supermode control, the next-generation, large-scale, high-efficiency $\mathrm{Si} / \mathrm{III}-\mathrm{V}$ hybrid circuitry will not be far away (see Fig. 3), and we expect a thorough revolution in the development of optical communications.

\section{REFERENCES}

[1] O. Boyraz and B. Jalali, "Demonstration of a silicon Raman laser," Opt. Express, vol. 12, pp. 5269-5273, Oct 2004.

[2] S. Lombardo, S. U. Campisano, G. N. van den Hoven, A. Cacciato, and A. Polman, "Room-temperature luminescence from Er-implanted semi-insulating polycrystalline silicon," Appl. Phys. Lett., vol. 63, pp. 1942-1944, Oct 1993.

[3] S. G. Cloutier, P. A. Kossyrev, and J. Xu, "Optical gain and stimulated emission in periodic nanopatterned crystalline silicon," Nat. Mater., vol. 4, pp. 887-891, Dec 2005.

[4] B. Kunert, S. Zinnkann, I. Németh, G. Lukin, R. Fritz, K. Volz et al., "Lasing of lattice-matched Ga(NAsP) quantum well heterostructures monolithically integrated on (001) Si substrate," in Proc. SPIE. vol. 7220, SPIE Photonics West 2009, San Jose, CA: SPIE, 2009, paper 7220-26.

[5] A. W. Fang, H. Park, O. Cohen, R. Jones, M. J. Paniccia, and J. E. Bowers, "Electrically pumped hybrid AlGaInAs-silicon evanescent laser," Opt. Express, vol. 14, pp. 9203-9210, Oct 2006.

[6] A. W. Fang, E. Lively, Y.-H. Kuo, D. Liang, and J. E. Bowers, "A distributed feedback silicon evanescent laser," Opt. Express, vol. 16, pp. 4413-4419, Mar 2008.

[7] A. Yariv, Optical Electronics in Modern Communications, 5th ed. New York: Oxford Univ. Press, 1997. 